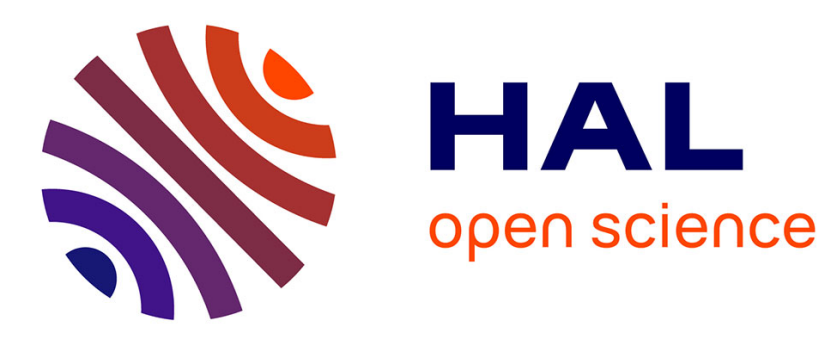

\title{
Improving antenna gain estimations in non-ideal test sites with auto-tunable filters
}

Artur Nogueira de Sao Jose, Virginie Deniau, Ursula Do Carmo Resende, Ricardo Adriano

\section{- To cite this version:}

Artur Nogueira de Sao Jose, Virginie Deniau, Ursula Do Carmo Resende, Ricardo Adriano. Improving antenna gain estimations in non-ideal test sites with auto-tunable filters. Measurement - Journal of the International Measurement Confederation (IMEKO), 2020, 159, pp1-9. 10.1016/j.measurement.2020.107720 . hal-02617160

\section{HAL Id: hal-02617160 \\ https://hal.science/hal-02617160}

Submitted on 25 May 2020

HAL is a multi-disciplinary open access archive for the deposit and dissemination of scientific research documents, whether they are published or not. The documents may come from teaching and research institutions in France or abroad, or from public or private research centers.
L'archive ouverte pluridisciplinaire HAL, est destinée au dépôt et à la diffusion de documents scientifiques de niveau recherche, publiés ou non, émanant des établissements d'enseignement et de recherche français ou étrangers, des laboratoires publics ou privés. 


\title{
Improving antenna gain estimations in non-ideal test sites with auto-tunable filters
}

Artur N. de São José, UFMG ${ }^{1}$, Virginie Deniau, IFSTTAR ${ }^{1}$, Úrsula do C. Resende, CEFET-MG ${ }^{1}$, Ricardo Adriano, UFMG ${ }^{1}$

\begin{abstract}
Estimating the gain of an antenna prototype is an important step during both design and validation phases. Nevertheless, many small technology companies and universities are not provided with adequate infrastructure to run this type of test. In this paper, we propose a computational tool to deal with undesirable contributions coming from non-ideal test conditions over electromagnetic measurements. It is based on auto-tunable filters and therefore it allows a lab technician to run the test without a major knowledge on signal processing. After a series of evaluations based on lab measurements, the proposed tool gives results as good as those provided by two other approaches but with a reduction of the total number of experimental runs. Furthermore, it is more efficient than the traditional time gating approach for low signal-to-noise ratio environments. Finally, a validation with different antennas and test sites confirmes its robustness.
\end{abstract}

Keywords: adaptive filtering, time gating, antenna, gain, calibration, OATS

2010 MSC: 00-01, 99-00

\section{Introduction}

Antenna calibration is a necessary procedure for manufacturers in order to check if a prototype or the final version of their product follows the design specifications. This type of evaluation requires specific test facilities, which

5 usually have high implementation costs and therefore it is possible that they are not numerous around a given locality. Consequently, if the regional laboratory 
equipment is not sufficient for the local demand, companies have to pay to use facilities in distant places or even abroad [1].

Test standards such as IEEE 149 [2] and IEEE C63.5-2017 [3] serve as guidelines for the calibration procedures of general-purpose and electromagnetic compatibility (EMC) antennas, respectively. In general, these standards define two possible test environments: a shielded room with internal electromagnetic (EM) absorbers and open area test sites (OATS). The first requirement can be achieved with a fully or a semi-anechoic chamber. Even though, this environment in terms of EM isolation is the best solution, it remains the most expensive one. For such a reason, antennas and radiofrequency devices manufacturers can prefer OATS tests for validation due to their limited resources. Nevertheless, measurements in open areas can present non-reliable results if the test site is affected by external electromagnetic noise and multipath sources.

Despite the existence of specific standards for tests in OATS, EM measurements in such environment are still topics of interest for the researchers. Recent investigations concern the characterization of the EM emissions of devices under test (DUT) in OATS. Indeed, it presents two main constraints. First, the traditional data acquisition in the frequency domain can demand a considerably long time to cover wide bands. Secondly, it requires a large isolated space, especially for DUTs with great dimensions. In order to address these issues, Arnd Frech [4, 5] proposes a real-time acquisition system that operates in the time domain. On the other hand, Sheng [6] proposes to split the wide band signal into several narrower band components through a wavelet transform unit. This leads to a parallel processing of the resulting components, which increases the overall efficiency of the signal processing structure. Similar approaches can be found on [7, 8. Finally, Mikulas Bittera 9] describes analytical expressions for the uncertainties inherent to the antenna calibration problem in OATS as well as techniques to minimize them.

$35 \quad$ Knowing that places to conduct ideal OATS tests are increasingly rare, this work proposes a filtering approach to enhance the quality of the antenna calibration performed in a non-ideal OATS. During this investigation, we considered 
the final user as a lab technician or engineer who does not necessarily have a signal processing background. For such a reason, auxiliary routines that automatically adjust the filters parameters were developed and incorporated. Thus, the main contributions of this work, to be further detailed, are:

1. an evidence that in low signal-to-noise (SNR) environments, the classical EMC solution provided by commercial vector network analyzers (VNAs) for noisy measurements, time gating, is strongly dependent on an uncontrollable variable (the phase of the interfering signal) and its performance can be non-satisfactory;

2. a new fitness function for the parameter selection mechanism described in 6] for adaptive filters and;

3. an automatic design algorithm for the time gating window, in opposition to the traditional manual adjustment provided by commercial VNAs.

\section{Test setup}

There are a few techniques for standardized antennas calibration. In this work, the standard site method (SSM) is used as a reference. Test setup includes a pair of antennas, one of them being the antenna under test (AUT), $d$ meters

55 apart from each other and $h_{1}, h_{2}$ meters respectively above a ground plane [10. In order to evaluate the performance of the proposed techniques, the AUT and the auxiliary antenna are connected to the two ports of a VNA. The VNA allows to characterize the properties of different devices by measuring input-output power ratios. In this work, we use the VNA to measure the $S_{12}$ transmission parameter of the aerial link and subsequently estimate, through the Friis equation, the AUT's gain over its operating frequency band[11. During the experiments, each measurement was preceded by a cable calibration procedure by using the VNA supplier reference calibration kit. The aim is to minimize the cables effects over the $S_{12}$ measurements.

To conduct this work, two measurement campaigns have been run: one at the Federal University of Minas Gerais (UFMG), Brazil and the other one at 
the French Institute of Science and Technology for Transport, Development and Networks (IFSTTAR), France. In order to facilitate the identification of the test setups described along this paper, we adopt the notations shown in Table 70 1. Figure 1 shows the experimental test setups of this study. Figures 1 a and $1 \mathrm{~b}$ represent the EM tests in non-ideal environments while Figs. $1 \mathrm{c}$ and $1 \mathrm{~d}$ show the test configurations in anechoic chambers. Since Lab1 and Lab2 were not designed for OATS tests, they contain various objects of different materials, shapes and sizes. Therefore, these objects can induce multipath and affect the $S_{12}$ measurements. These effects are potentially stronger for the non-directive antennas. Furthermore, the presence of communication devices like routers and terminals connected to WiFi networks surrounding the test site can also affect the accuracy of the characterization tests. Finally, the main characteristics of the four AUTs used in this study are summarized in Table 2, These antennas are referenced by their corresponding labels in the first column of this table.

Table 1: Test facilities used for the experiments.

\begin{tabular}{||ccc||}
\hline Notation & Facility & Institution \\
\hline \hline Lab1 & Applied Electromagnetics Lab & UFMG \\
\hline Lab2 & Electromagnetic Compatibility Lab & IFSTTAR \\
\hline Cha1 & Anechoic Chamber & UFMG \\
\hline Cha2 & Anechoic Chamber & IFSTTAR \\
\hline
\end{tabular}

Table 2: Antennas used during this investigation, in terms of notations, some properties and references for more details.

\begin{tabular}{||cccc||}
\hline Notation & Directivity & Range & Reference \\
\hline \hline AUT1 & Directive & $1.5-4.5 \mathrm{GHz}$ & {$[12]$} \\
\hline AUT2 & Omnidirectional & $1.5-4.5 \mathrm{GHz}$ & {$[12]$} \\
\hline AUT3 & Directive & $700 \mathrm{MHz}-18 \mathrm{GHz}$ & {$[13]$} \\
\hline
\end{tabular}




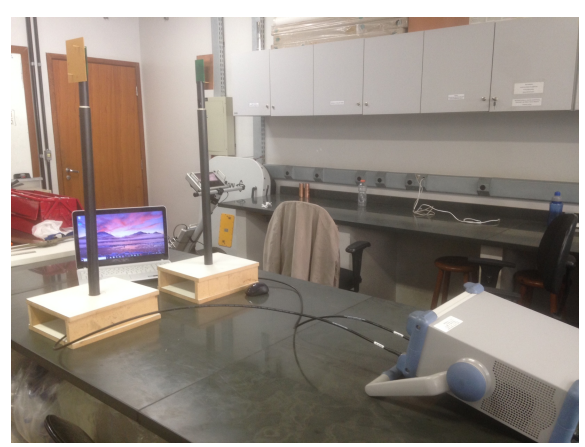

(a) Lab1

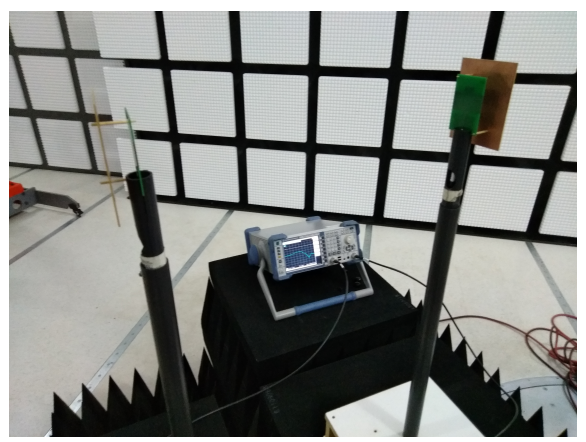

(c) Cha1

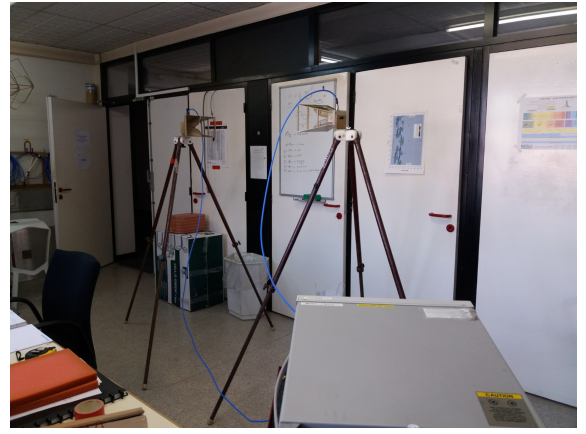

(b) Lab2

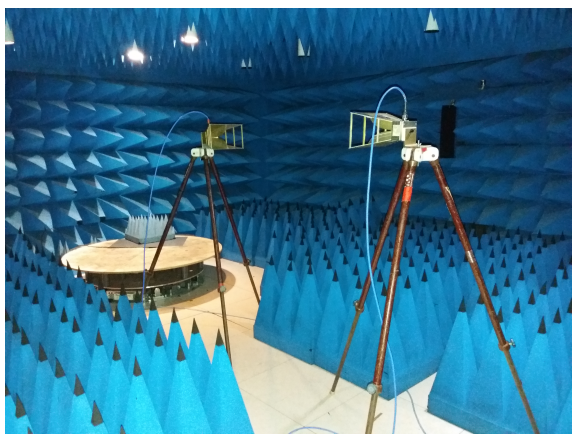

(d) Cha2

Figure 1: Test setups mounted for this investigation.

\section{Potential limitations of the existent solutions}

This section presents some state of the art techniques used to deal with interferences and other undesirable effects over electromagnetic measurements, as well as their potential flaws.

\subsection{Time gating}

A common technique used in EMC labs to deal with test environment spurious effects is time gating [14]. These are filters designed over the time domain in an attempt to reject any contributions outside a certain interval through a window function. Most modern VNAs offer such a feature but window type,

90 location and duration adjustments are usually manual. 
Such a method can present potential failures. Indeed, some interfering signals can be intermittent and dependent on the configuration, location and quantity of sources (in this case, WiFi routers) near the test setup. Consequently, the time instant in which they appear, their duration and intensity are all unpredictable but, nevertheless, TG has to present good responses. To consider this potential variability, we artificially added a $2.4 \mathrm{GHz}$ phasor, with variable amplitude and phase, to the original measurements and further evaluated the filter performance. Figure 2a shows the TG responses for a signal that results from the complex sum of a $S_{12}$ measurement taken at Lab1 (gray curve) and an unitary phasor with variable phase. That means that we did not introduce artificial modification of the EMI amplitude; we only consider the phase impact. The filtered curves are smooth and the differences between them are negligible, which indicates that the state-of-the-art solution is suitable in this case. However, if we increase 5 or 10 times (see Figs. 2b and 2c) the amplitude of the narrow-band interference, not only are there significant differences between the curves but also, but none of them is satisfactory. The significant differences between the curves highlights that the TG performance depends upon the EMI phase which is an uncontrollable variable. We then selected the worst curve from Fig. 2c (see Fig. 2d) to assess if the proposed technique is able to retrieve the original data.

\section{2. $D A E-1$ and $D A E-2$}

Two recent alternate approaches for antenna calibration in non-ideal test conditions propose the incorporation of design and analysis of experiments (DAE) principles [15]. These approaches are described in [11, 16] and will 115 be hereinafter called DAE-1 and DAE-2, respectively. DAE-1 and DAE-2 are based on spatial rearrangements of the test setup, data extraction from replicated measurements and further mathematical manipulations, mainly based on statistical averages.

Figure 3 illustrates the measurements taken for the further processing via

DAE-1 and DAE-2. These measurements, performed with AUT1 in Lab1 by 


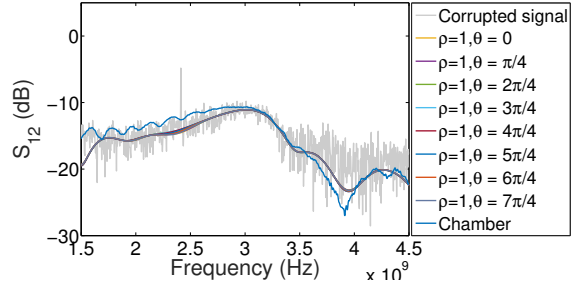

(a)

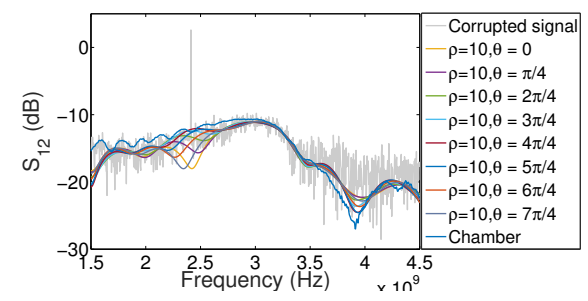

(c)

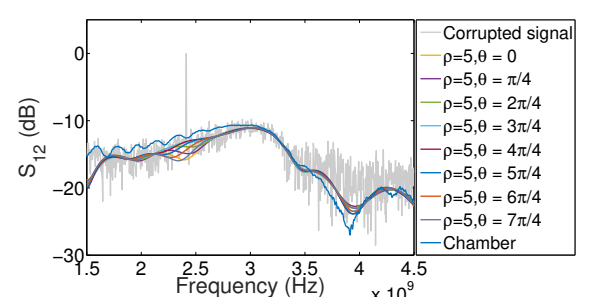

(b)

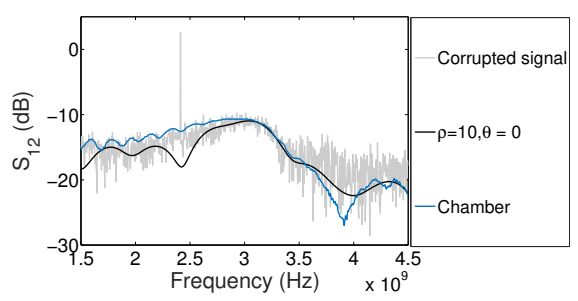

(d)

Figure 2: Manually adjusted time gating applied to the noisy $S_{12}$ signal added to a 2.4 $\mathrm{GHz}$ phasor with variable amplitude $(\rho)$ and phase $(\theta)$.

using a VNA with internal power equal to $-50 \mathrm{dBm}$, contain actual $S_{12}$ curves embedded with spurious contributions - mainly thermal noise. The legends from Fig. 3 follow this definition: height of the antennas, rotation angle applied to the test setup (spatial rearrangements) and a label for each sampled waveform taken from a given configuration. This figure does not contain all the test runs (10 samples $\times 4$ configurations $=40$ runs), but it is sufficient to evidence the high amount of time required by the mentioned approaches. Evidently, a lower number of runs could be used, but resulting in degraded DAE-1 and DAE-2 performances.

\subsection{Adaptive Filtering}

Adaptive filters can be described as variable transfer functions defined in the Z-Transform domain, $H(z)$ [17. Their use for emissions measurement improvements in OATS and antenna design has been recently documented [6, 5, 18. In 


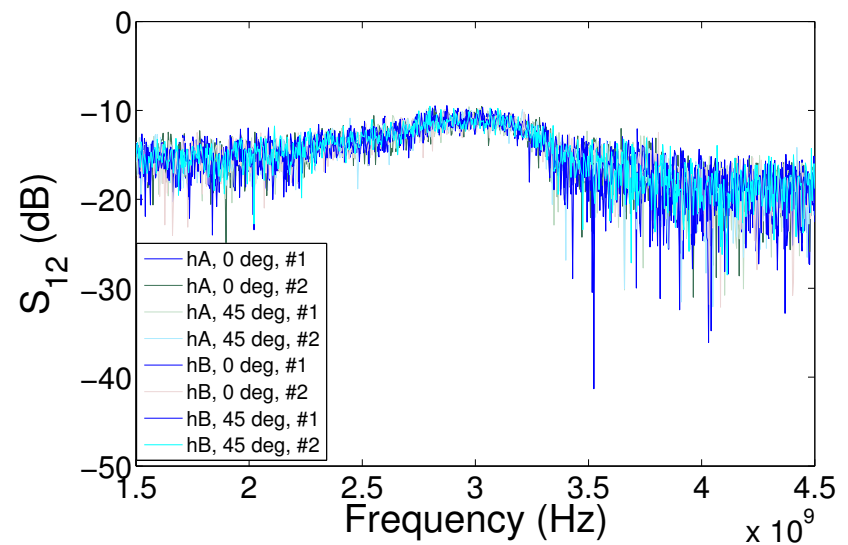

Figure 3: Some measurements taken for further processing via DAE-1 and DAE-2.

opposition to traditional filters, they include auto-adjustment mechanisms to fit signals.

One important aspect of an adaptive filter design concerns the definition of an adaptive algorithm and its parameters specification. In this work, we adopt the normalized least mean squares (NLMS) algorithm, which is described by the following iterative process:

$$
h[n+1]=h[n]-\mu \hat{\nabla} E\left(e^{2}[n]\right),
$$

where $h[n+1]$ is the filter impulse response evaluated at the discrete time index $n+1$ ( $0 \leq n \leq M-1, M$ being the signal duration), $\mu=\mu_{0} /\|u[n]\|^{2}$ (where $0<\mu_{0}<1$ is a constant and $u[n]$ is the noise floor) is called step-size, $E($. is the expectation operator, $e[n]$ is called error function, $E\left(e^{2}[n]\right)$ is the mean squared error (MSE) and $\hat{\nabla} E\left(e^{2}[n]\right)$ is an estimate of the associated gradient function. Here, $e[n]$ represents the difference between the noisy signal and an estimation of the actual noise provided by the filter. With an accurate filter design, $e[n]$ can provide a correct representation of the actual signal once the iterative process from Eq. 1 has converged. 
$\mu_{0}$ and for the impulse response vector length, $L$ (also known as the filter order), as well. These parameters, usually defined through empirical knowledge, significantly impact the dynamics and accuracy of the process described in Eq. 1. In order to prove the importance of an accurate filter design, we define the following function, which is inversely proportional to the filter performance:

$$
f=\left(\mathbf{E}_{i}-\mathbf{E}_{\text {ref }}\right)\left(\mathbf{E}_{i}-\mathbf{E}_{\text {ref }}\right)^{H}+\left.0.1\left(\mathbf{E}_{i}-\mathbf{E}_{\text {ref }}\right)\left(\mathbf{E}_{i}-\mathbf{E}_{\mathbf{r e f}}\right)^{H}\right|_{2.4 G H z},
$$

where $\mathbf{E}_{i}$ and $\mathbf{E}_{r e f}$ are, respectively, the $i^{t h}$ candidate error curve (obtained from the steady-state response of the $(\mu, L)_{i}$ filter) and the reference, both in the frequency domain; $(.)^{H}$ is the Hermitian operator applied to a vector (i.e. its conjugate transpose) and (.) $\left.\right|_{2.4 G H z}$ means evaluation only at $2.4 \mathrm{GHz}$. We then define the ranges $0 \leq L \leq 100,0 \leq \mu_{0} \leq 0.1$ and evaluate Eq. 2 as shown in Fig. 4. This graphic demonstrates the filter sensitivity to the parameters selection. As an example, if we move from $(0.014,33)$ to $(0.099,10)$ in the $(\mu, L)$ plane, the function described in Eq. 2 increases about 4 times.

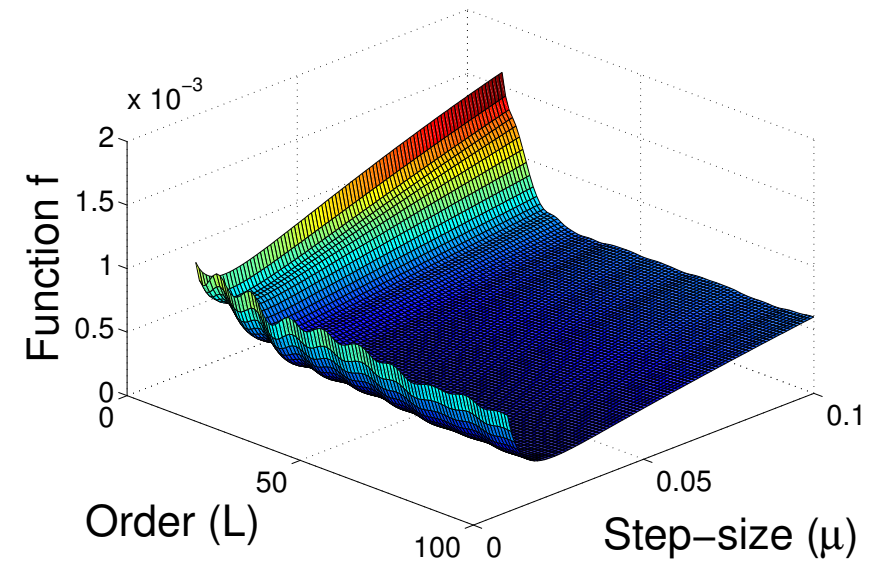

Figure 4: Filter performance as a function of its parameters. Low values: good performance; high values: poor performance. 


\subsection{Summary}

165 straints found in the state-of-the-art. Such limitations reinforce the need for more robust techniques.

Table 3: State of the art approaches and their constraints.

\begin{tabular}{||cc||}
\hline Technique & Constraint \\
\hline \hline Time gating & Low SNR \\
\hline DAE-1 and DAE-2 & Time consuming \\
\hline Adaptive filtering & Multipath and high sensitivity to the parameters selection \\
\hline
\end{tabular}

\section{Methodology}

The structure of the proposed tool is based on a series connection of two discrete-time filters: adaptive filter and time gating. Such a cascade structure was finally defined after exhaustive unsuccessful trials : first, with adaptive filters only, then with time gating only and finally, an inverted cascade connection, i.e. TG before AF. Figure 5 summarizes the arrangement and the main signals involved. This diagram also shows two blocks named PSO and window design.

175 These elements, to be further detailed, allow auto-adjustment mechanisms. In the following sections of this paper, PSO and window design are mentioned as the auxiliary algorithms in contrast with the adaptive filter and time gating stages, which are the main algorithms. Their interactions with the filters are symbolized in Fig. 5 through a set of switches opened by default and closed only during the parameters selection. Then, the input signals can be processed and a filtered version of the $S_{12}$ signal is produced.

\subsection{Stage 1}

The pre-configuration of the adaptive filter has to take into account a significant amount of candidate solutions within the $(\mu, L)$ plane. The reason is that 


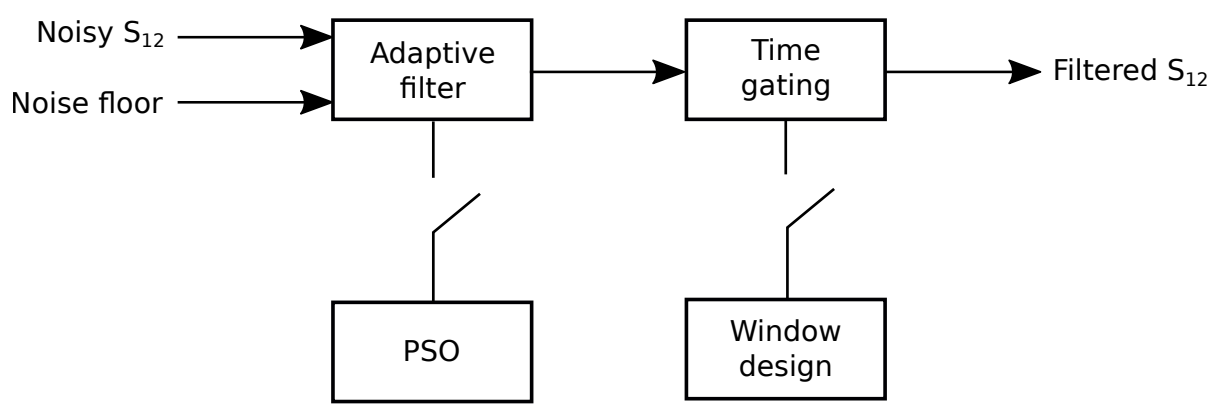

Figure 5: Proposed structure: a series connection of an adaptive filter and time gating, both pre-configured through the PSO and window design algorithms, respectively.

no previous empirical knowledge concerning this choice is available. Hence, all regions of the search space are equally probable to contain the optimum solution. In such a scenario, population-based optimization algorithms tend to be more efficient than gradient-based ones.

In a recent paper about the use of AFs on an OATS EMC test called radiated emissions, Sheng et al [6] propose a configuration based on the particle swarm optimization (PSO) method. In fact, the PSO is being increasingly applied to AF problems [19, 20, 21] in a variety of ways. In this work, we apply the PSO to find the most suitable parameters for the AF by means of a new fitness function.

The PSO is an evolutionary optimization algorithm which works inside a search space; in this case, the $(\mu, L)$ plane. In order to evaluate the pertinence of each candidate solution (the particles) and select the best ones, a fitness function is defined and further minimized. This function is usually inversely proportional to the particle performance. In this work, the fitness function is a certain function of the AF output, also known as the error signal, $e[n]$. In [6], the fitness function is the mean squared error, MSE [6]. In other words, it prioritizes candidate solutions that produce filtered signals with lower energy levels. This is a good approach when one can ensure that, given a noisy measurement, the filter only extracts undesirable contributions while keeping the useful signal. In this case, the most suitable filter is the one that reduces more the energy levels 205 of the resulting signal. However, during this investigation, we identified $(\mu, L)$ 
parameters, provided by the PSO, that filter the noise but also affect the useful signal. This led us to the formulation of a different approach.

In this work, we propose the following fitness function:

$$
f=\rho(e[n], u[n]),
$$

where $\rho(.,$.$) is the Pearson correlation coefficient, defined as$

$$
\rho(X, Y)=\operatorname{cov}(X, Y) / \rho_{X} \rho_{Y}
$$

$22510 \leq L \leq 50$ the limits of the search space. Concerning the inertial weight, we evaluated both functions defined above. The decreasing and increasing profiles lead, respectively, to growing and decaying velocities. The first one allows a 
good exploitation of the search space, avoiding premature convergences to local minimum but requires more iterations. The second one allows faster convergence rates but with higher probability of falling into a local minimum. Besides this, we used an approach based on sub-swarms, as described by F. Marini and B. Walczak in [22], in order to increase diversity during the search for the optimum solution.

\subsection{Stage 2}

In the second stage, TG is used in order to minimize multipath and thermal noise, considering that EMIs were previously mitigated by the adaptive filter. The novelty here is the presence of the auxiliary routine named window design in figure 5. It automatically sets the location and duration of the time domain window. To do so, it first extracts the signal envelope, by calculating the corresponding Hilbert transform and then its absolute values. After that, the routine identifies the peak value, from which it calculates four other points defining the time domain window: (i) the beginning and (ii) the end of the rise time, as well as the beginning (iii) and (iv) the end of the fall time.

Between (i) and (ii), the rising portion of a Blackman function is used; between (ii) and (iii), we use the unitary portion of the rectangular window and, finally, between (iii) and (iv), we apply the falling portion of the Hamming window. We defined (i) and (iv) as the time instants correspondent to $5 \%$ of the peak value and (ii) and (iii) as $40 \%$. These levels are a good compromise considering the time domain response of AUT1, AUT2 and AUT3 and therefore they can potentially cover different types of antennas. Figure 6 illustrates the automatic window applied to the AUT3 $S_{12}$ signal in the time domain, after the first stage (AF).

\section{Results}

The results here exposed were obtained from different test conditions, in 255 order to estimate the working range of each evaluated approach. For the measurements involving AUT1 and AUT2, the VNA power was set to -50 dBm, while 


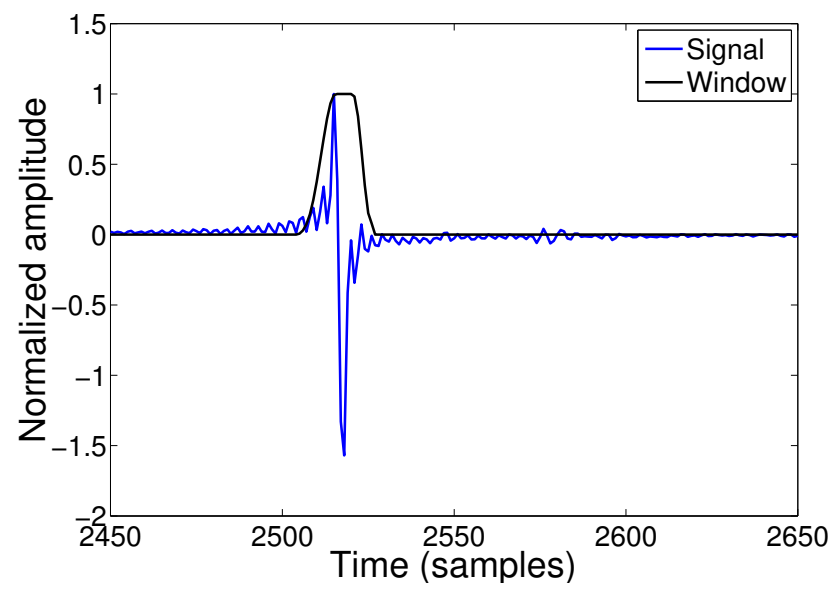

Figure 6: Automatic TG window applied to the AUT3 $S_{12}$ signal in the time domain, after the first stage $(\mathrm{AF})$.

for AUT3 the power was $-30 \mathrm{dBm}$. In addition, the test setup was brought to the vicinity of a WiFi router. Additionally, the interference levels were artificially increased up to 10 times. The last procedure aims to evaluate the performance of the proposed structure in a scenario where TG with the traditional manual adjust can fail, as discussed in Section 3.1. Finally, the different directivities of the antennas in Table 2 allow us to evaluate the methodology under different multipath levels, due to omnidirectional antennas producing more reflections than directive ones. All the following results are expressed through gain versus frequency curves, obtained by applying the Friis equation to the $S_{12}$ curves and with $1 \mathrm{~m}$ distance between the antennas.

Figure 7 concerns the configuration with AUT1 and without any change on the original interference levels. It compares the results of both the proposed filtering approach and the DAE-based techniques. The purple curve, obtained in Cha1, is the reference result and the gray one is relative to the $S_{12}$ measurements carried out at Lab1 nearby a WiFi router. The gray curve evidences a narrow-band EMI coming from this device near $2.4 \mathrm{GHz}$. All approaches lead to a minimization of both the EMI energy and the oscillations present on the measurement, which are mainly caused by the thermal noise coming from the 
VNA operation in $-50 \mathrm{dBm}$. Nevertheless, there is a loss of performance around $4 \mathrm{GHz}$, especially for the DAE-based methods. This is caused by the strong negative peaks shown in Fig. 3, which are completely random and therefore cannot be easily eliminated through AF or DAE-1/DAE-2. In this scenario, TG decreases the thermal noise and consequently the black curve from Fig. 3 is closer to the chamber graph than the others are. Therefore, the performances of all methods are satisfactory for frequencies up to $3 \mathrm{GHz}$ and, for the $3-4.5 \mathrm{GHz}$ range, there is a performance degradation which is stronger for DAE-1/DAE-2.

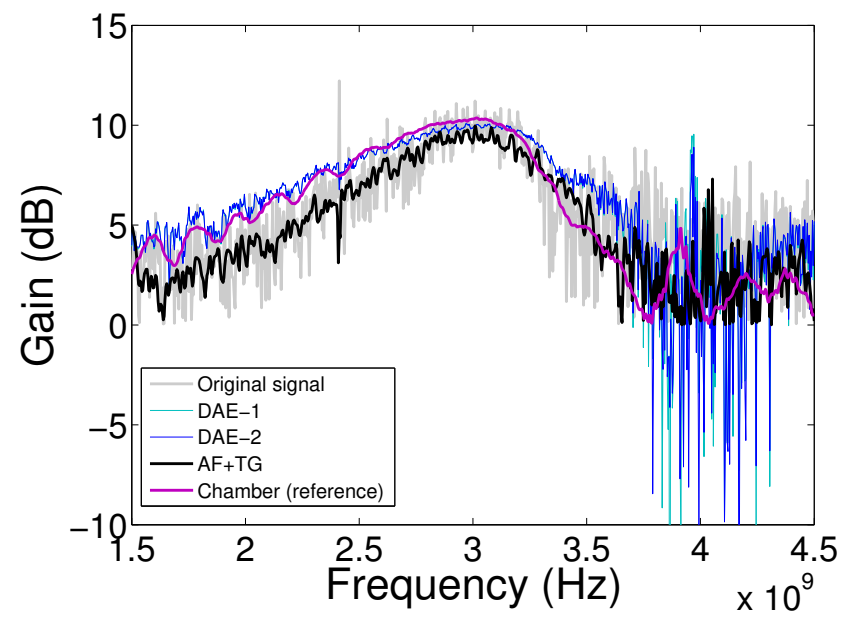

Figure 7: Retrieved gain curves.

After increasing 10 times the WiFi noise level, we evaluated the performance of the AF+TG cascade structure under two different configurations. The first one uses the PSO with the proposed fitness function, described in Eq. 3, while the second one employs the classical MSE function. Table 4 summarizes the resulting $(\mu, L)$ parameters.

Results obtained for the 6 configurations of Table 4 are exposed in Figs. 8, 10 through gain curves. These figures expose the reference chamber measurement, the noisy signal, and the outputs of the first and second stages of the cascade structure from Fig. 5. They contain both a complete view of the curves and a zoomed one, focused on the $2.4 \mathrm{GHz}$ EMI frequency band. Figure 8 presents 
Table 4: $(\mu, L)$ parameters obtained through PSO with different fitness functions.

\begin{tabular}{||ccc||}
\hline Antenna & Classical fitness & Proposed fitness \\
\hline \hline AUT1 & $(0.1,49)$ & $(0.0456,27)$ \\
\hline AUT2 & $(0.1,50)$ & $(0.01,48)$ \\
\hline AUT3 & $(0.1,47)$ & $(0.0151,13)$ \\
\hline
\end{tabular}

the results for the directive AUT1 antenna. For this case, the proposed fitness leads to a less smooth curve when compared to the other one obtained with the classical approach, but with the advantage of reducing more the EMI and distorting less the adjacent frequencies. Figure 9, which concerns the omnidirectional AUT2 antenna, evidences a good performance of both approaches. Finally, Fig. 10, which concerns a horn directive antenna, shows a considerably better performance with the proposed fitness function for the AF approach. In this case, the poor design of the AF generated by means of the MSE fitness was compensated by an efficient automatic design of the TG window. Consequently the overall performances achieved the same good level.

In order to verify the consistency of the parameter values exposed in Table 4. we ran 10 times the PSO routine per fitness function, and having the AUT1 signal as input for the filtering structure. Indeed, given the non-deterministic nature of the PSO, variations of the parameter values are expected. Table 5 shows these parameters, as well as the corresponding Pearson correlation coefficients between the filtered and reference anechoic chamber curves. The dispersion levels are higher for the proposed fitness function, but the correlation levels are, in average, higher than those obtained with the classical approach (0.8600 against 0.8142$)$.

\section{Conclusions}

This work proposes a new signal processing technique for antenna gain estimations in non-ideal open area test sites. The proposed structure combines 


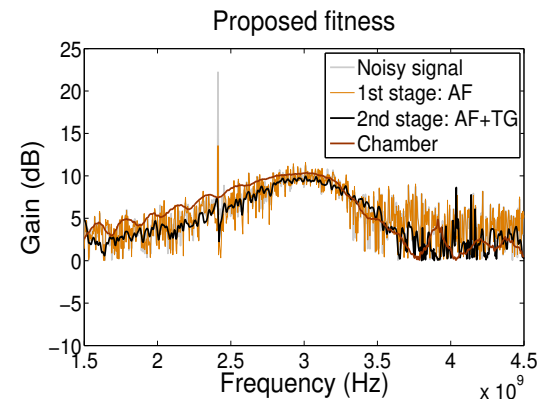

(a)

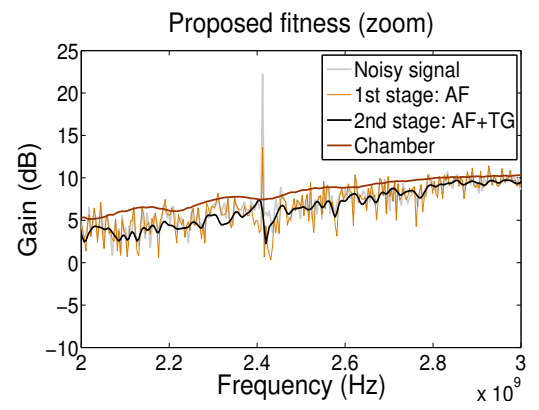

(c)

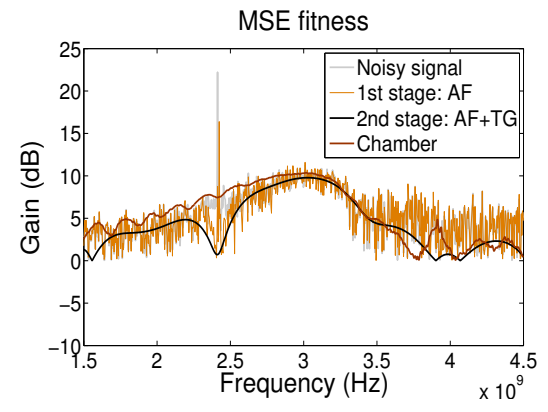

(b)

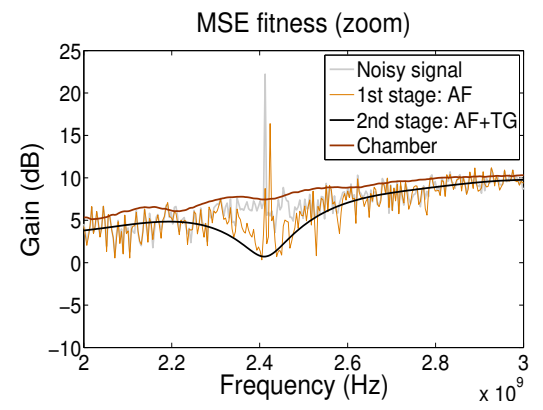

(d)

Figure 8: AUT1 gain curves revealing the performance of the proposed signal processing structure. (a) and (c) PSO with the proposed fitness. (b) and (d) PSO with the classical MSE fitness.

classical techniques and includes auxiliary algorithms, i.e. the PSO and the window design routines, in order to fit with the EMC problems. These auxiliary routines automatically determine the optimal $(\mu, L)$ parameters for the adaptive filter and the location and duration of the time gating window. Therefore, the general contribution of this work is to shorten the bridge between the fields of signal processing and electromagnetic compatibility. Many interesting signal processing developments are described in the specialized literature without the compromise of fitting in a specific application domain. Hence, the penetration of these advances over the EMC community (in both industrial and academic sectors) is not so high. Thanks to the development of automatic algorithms 


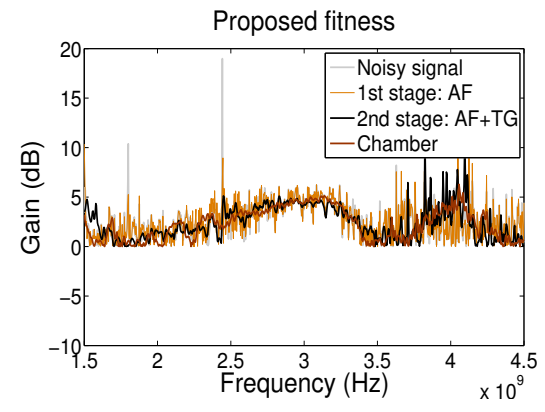

(a)

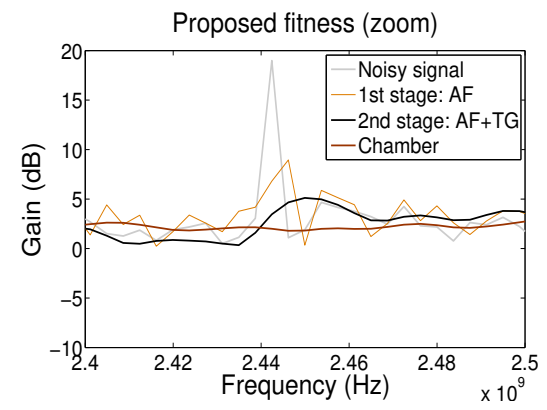

(c)

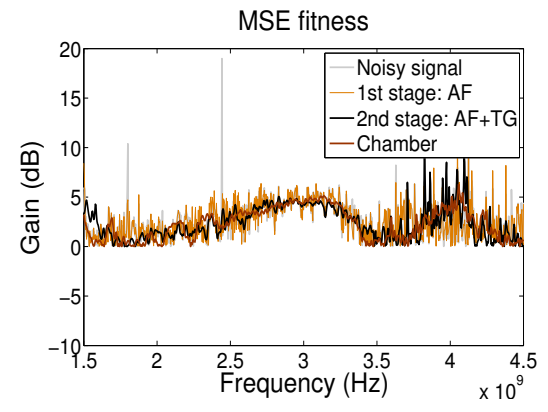

(b)

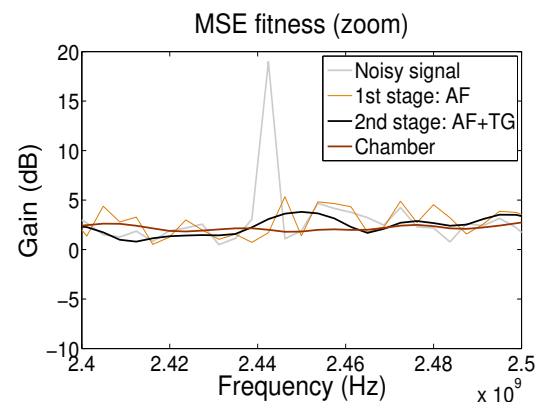

(d)

Figure 9: AUT2 gain curves revealing the performance of the proposed signal processing structure. (a) PSO with the proposed fitness. (b) PSO with the classical MSE fitness.

to adjust the filters, lab technicians or engineers without a deep knowledge on signal processing can use the proposed technique.

This work also highlights that time gating is strongly dependent on an uncontrollable variable (phase of the interfering signal), especially in low SNR environments. In such a situation, the new fitness function for the PSO algorithm can improve the results. Its effectiveness was compared to the classical MSE function efficiency. Indeed, the calculation of correlation levels between the filtered curves and reference anechoic chamber measurements showed that the proposed fitness provides on average better results than the MSE function.

Moreover, the proposed signal processing structure reduces the number of experimental runs, and therefore the total test time, in relation to DAE-1 and 


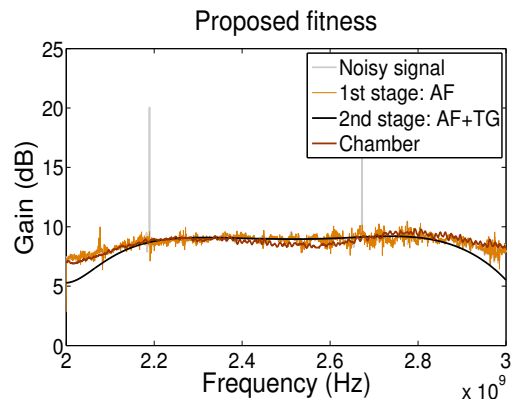

(a)

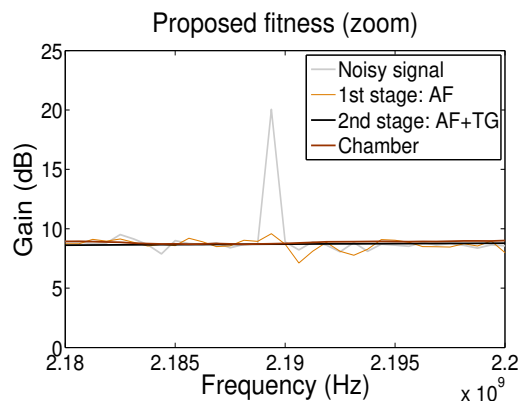

(c)

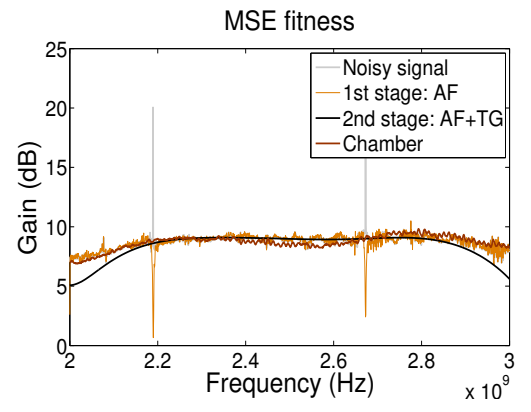

(b)

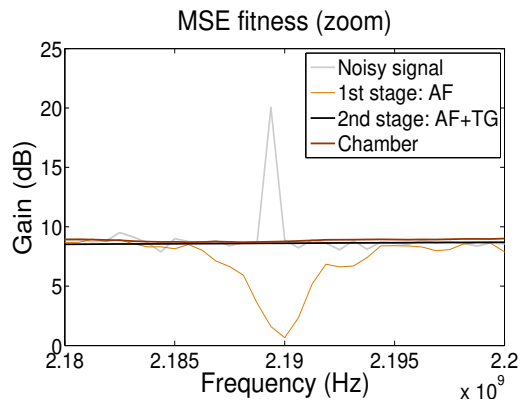

(d)

Figure 10: AUT3 gain curves revealing the performance of the proposed signal processing structure. (a) PSO with the proposed fitness. (b) PSO with the classical MSE fitness.

DAE-2. While DAE-1 and DAE-2 can require a great amount of experimental runs, our approach only requires two measurements: the corrupted signal and a single noise floor sample.

In conclusion, this work demonstrated the advantages of the proposed methodology when compared to other techniques. The first advantage is the reduction of the required test duration. The second advantage is the new fitness function to configure a PSO routine that sets the adaptive filter parameters. Finally, we demonstrated that our technique can be an alternative for the classical time gating approach, which can fail in low SNR environments. 
Table 5: $(\mu, L)$ parameters obtained after 10 PSO simulations with both classical and proposed fitness functions and the corresponding correlation between filtered and chamber curves.

\begin{tabular}{ccccccc}
\hline \multicolumn{4}{c}{ Classical fitness } & \multicolumn{4}{c}{ Proposed fitness } & Inertial weight \\
\hline$\mu$ & $\mathrm{L}$ & Correlation & $\mu$ & $\mathrm{L}$ & Correlation & - \\
0.1 & 50 & 0.8518 & 0.04153 & 22 & 0.8746 & Decreasing \\
0.1 & 46 & 0.7941 & 0.05630 & 13 & 0.8477 & Decreasing \\
0.1 & 46 & 0.7941 & 0.04561 & 27 & 0.8703 & Decreasing \\
0.1 & 44 & 0.8020 & 0.04561 & 27 & 0.8703 & Decreasing \\
0.1 & 42 & 0.8213 & 0.03056 & 15 & 0.8412 & Decreasing \\
0.1 & 49 & 0.8518 & 0.03479 & 14 & 0.8385 & Increasing \\
0.1 & 48 & 0.7876 & 0.04220 & 22 & 0.8746 & Increasing \\
0.09890 & 46 & 0.7953 & 0.04220 & 22 & 0.8746 & Increasing \\
0.1 & 50 & 0.8518 & 0.07354 & 11 & 0.8541 & Increasing \\
0.09970 & 47 & 0.7921 & 0.07095 & 11 & 0.8540 & Increasing \\
\hline Average correlation & 0.8142 & $\ldots$ & - & 0.8600 & \\
\hline
\end{tabular}

\section{Acknowledgements}

This study was financed in part by the Coordenação de Aperfeiçoamento de Pessoal de Nível Superior - Brasil (CAPES) - Finance Code 001, FAPEMIG and IFSTTAR. The authors would like to acknowledge Dr. Jonathan Villain (IFSTTAR) and Prof. Lucas Batista (DEE/UFMG) for the very productive discussions about the PSO algorithm.

\section{References}

[1] A. N. de São José, U. C. Resende, M. A. de Menezes, J. H. Ferreira, Proposal for a brazilian regulation of electromagnetic compatibility applied to automotive vehicles, in: 2016 IEEE International Symposium on Electro- 
magnetic Compatibility (EMC), 2016, pp. 495-500. doi:10.1109/ISEMC. 2016.7571698 .

[2] Ieee standard test procedures for antennas, ANSI/IEEE Std 149-1979.

[3] American national standard for electromagnetic compatibility-radiated emission measurements in electromagnetic interference (emi) controlcalibration and qualification of antennas (9 khz to $40 \mathrm{ghz}$ ), ANSI C63.52017 (Revision of ANSI C63.5-2005) (2017) 1-114doi:10.1109/IEEESTD. 2017.7920447

[4] A. Frech, P. Russer, Real-time ambient noise cancellation for emi measurements on open area test sites, in: 2012 Asia-Pacific Symposium on Electromagnetic Compatibility, 2012, pp. 213-216. doi:10.1109/APEMC. 2012.6238010

[5] A. Frech, A. Zakaria, S. Braun, P. Russer, Ambient noise cancelation with a time-domain emi measurement system using adaptive filtering, in: 2008 Asia-Pacific Symposium on Electromagnetic Compatibility and 19th International Zurich Symposium on Electromagnetic Compatibility, 2008, pp. 534-537. doi:10.1109/APEMC.2008.4559930

[6] L. Sheng, W. Bangmin, Z. Lanyong, Intelligent adaptive filtering algorithm for electromagnetic-radiation field testing, IEEE Transactions on 【 Electromagnetic Compatibility 59 (6) (2017) 1765-1780. doi:10.1109/ 375 TEMC.2017.2656896.

n [7] F. G. Awan, A. Kiran, Cancellation of interference for emission measurement in open area test site, Measurement 111 (2017) 183 - 196. doi:https://doi.org/10.1016/j.measurement.2017.07.037.

口 URL http://www.sciencedirect.com/science/article/pii/ 380 S0263224117304736

口 [8] F. G. Awan, N. M. Sheikh, Model for radiation emission emc measurement at oats: Issues and approaches, Measurement 42 (7) (2009) 1045 - 1052. 


doi:https://doi.org/10.1016/j.measurement.2009.03.008,
URL http://www.sciencedirect.com/science/article/pii/
S0263224109000566
घ9] M. Bittera, V. Smiesko, K. Kovac, Modified uncertainty estimation
of antenna factor measurement by standard site method, Measure-
ment $45(2)(2012) 190-198$, special issue on Electrical Instruments.
doi:https://doi.org/10.1016/j.measurement.2011.07.002,
URL http://www.sciencedirect.com/science/article/pii/
S026322411100220X

[10] V. Trainotti, Electromagnetic compatibility (emc) antenna gain and factor, IEEE Transactions on Electromagnetic Compatibility 59 (4) (2017) 10061015. doi:10.1109/TEMC.2016.2642833.

[11] A. N. de São José, M. V. A. Contreras, R. Adriano, U. d. C. Resende, J. F. Mologni, Uncertainties minimization in open environment antenna gain estimations, in: 2017 IEEE 3rd Global Electromagnetic Compatibility

\ Conference (GEMCCON), 2017, pp. 1-5. doi:10.1109/GEMCCON.2017. 8400666 .

[12] M. Africano, J. O. Vargas, R. Adriano, D. B. Oliveira, A. C. Lisboa, Ground penetrating radar antenna design for homogeneous and low-loss dielectric multilayer media, submitted to Journal of Microwaves, Optoelectronics and Electromagnetic Applications.

[13] I. A.H. Systems, Sas-571 double ridge guide horn antenna, https://www. ahsystems.com/datasheets/SAS-571_Horn_Antenna_Datasheet.pdf, access: 10/31/2019 (2019).

[14] J. Dunsmore, Gating effects in time domain transforms, in: 2008 72nd 口 ARFTG Microwave Measurement Symposium, 2008, pp. 1-8. doi:10. 1109/ARFTG. 2008.4804303. 
[15] D. C. Montgomery, Design and analysis of experiments, John wiley \& sons, 2017.

[16] S. M. Fróes, P. Corral, M. S. Novo, M. Aljaro, A. C. de C. Lima, Antenna radiation pattern measurement in a nonanechoic chamber, IEEE Antennas

n and Wireless Propagation Letters 18 (2) (2019) 383-386. doi:10.1109/ LAWP.2019.2891972

[17] A. Sayed, Adaptive Filters, Wiley - IEEE, Wiley, 2011. URL https://books.google.fr/books?id=VBaenqIVftUC

[18] S. Lu, J. Sun, G. Wang, J. Tian, A novel gsc beamformer using a combination of two adaptive filters for smart antenna array, IEEE Antennas and Wireless Propagation Letters 11 (2012) 377-380. doi:10.1109/LAWP. 2012.2192250

[19] N. K. Rout, D. P. Das, G. Panda, Particle swarm optimization based active noise control algorithm without secondary path identification, IEEE Transactions on Instrumentation and Measurement 61 (2) (2012) 554-563. doi:10.1109/TIM.2011.2169180.

[20] K. Basu, S. Nanda, An adaptive filtering technique with self-adaptive pso for estimation of non-stationary signals, in: 2016 International Conference on Communication and Signal Processing (ICCSP), 2016, pp. 1057-1061. doi:10.1109/ICCSP. 2016.7754311

${ }_{430}$ [21] D. J. Krusienski, W. K. Jenkins, A modified particle swarm optimization algorithm for adaptive filtering, in: 2006 IEEE International Symposium घ on Circuits and Systems (ISCAS), 2006, pp. 4 pp.-140. doi:10.1109/ ISCAS.2006.1692541

[22] F. Marini, B. Walczak, Particle swarm optimization (pso). a tutorial, Chemometrics and Intelligent Laboratory Systems 149 (2015) 153 - 165. doi:https://doi.org/10.1016/j.chemolab.2015.08.020 
S0169743915002117 